\title{
Laparoscopic Treatment of Isolated Spleen Metastases in Recurrent Ovarian Cancer
}

\author{
Shin SJ, Chung H, Kwon SH, Cha SD and Cho $\mathrm{CH}^{*}$ \\ Department of Obstetrics and Gynecology, School of Medicine, Keimyung \\ University, Daegu, Korea
}

Case Report

Volume 1 Issue 5

Received Date: October 31, 2016

Published Date: December 06, 2016

*Corresponding author: Chi-Heum Cho, Department of Obstetrics and Gynecology, School of Medicine, Keimyung University, 56 Dalseong-Ro, Jung-Ku, Daegu, South Korea, 700-712, Tel: 82-53-250-7518; E-mail: chcho@kmu.ac.kr

\section{Abstract}

Recurrence of epithelial ovarian cancer as widespread metastases throughout the peritoneum is often observed. We report three cases of isolated splenic metastasis as the first site of recurrence of ovarian cancer. Patients were treated with laparoscopic splenectomy followed by six cycles of platinum-based chemotherapy. Currently, they remain alive and have been disease free for at least 24 months since the last operation. We suggest that laparoscopic splenectomy is an effective therapeutic modality for isolated splenic metastasis in ovarian cancer.

Keywords: Ovarian cancer; Recurrence; Spleen metastasis; Laparoscopy

\section{Introduction}

Recurrence of ovarian cancer occurs via the peritoneum, which presents with multiple seedings and is commonly associated with peritoneal carcinomatosis. Unlike most other cancers, ovarian cancer rarely disseminates through the vasculature even though it may involve pelvic and/or paraaortic lymph nodes [1]. As a consequence of its pathophysiologic mechanism, isolated parenchymal metastasis of any organ in ovarian cancer is rare and this is particularly the case with isolated spleen metastasis. However, single organ metastasis, such as spleen, is often reported in other types of malignancies. The majority of splenic metastases arise from hematological malignancies, such as malignant lymphoma or leukemia. Secondary debulking may be considered as a treatment option since it may improve the prognosis when there is no sign of residual disease, but it will not be effective when there are multiple seedings or multiple organ metastases. However, it can be considered an effective treatment option only if there is a solitary lesion of recurrence. To our knowledge only 36 cases of isolated spleen parenchymal metastases have been reported. We report three cases of isolated splenic metastasis as the first site of recurrence of ovarian cancer and suggest laparoscopic splenectomy as an effective therapeutic modality for this type of metastasis in ovarian cancer.

\section{Cases}

\section{Case 1}

A 66-year-old woman was admitted to our department for evaluation of a splenic mass that was detected on positron emission tomography (PET). Three years prior to her admission, she was diagnosed with poorly differentiated neuroendocrine ovarian carcinoma, large cell stage IIIc (International Federation of Gynecology and Obstetrics [FIGO]). During her regular follow-up, a PET scan revealed a newly formed, large, well-defined, and round intense hypermetabolic lesion at the inverted 
splenic hilum (Figure 1) her serum carbohydrate antigen (CA)-125 value was $59.98 \mathrm{U} / \mathrm{ml}$ (normal range 0-35 $\mathrm{U} / \mathrm{ml}$ ). Since the spleen seemed to be the only site of ovarian metastasis, laparoscopic splenectomy was performed by a gynecologic oncologist. The patient was discharged on postoperative day 6 without any postoperative complications. Macroscopically, the spleen weighed $200 \mathrm{~g}$ and measured $9.6 \times 6.0 \times 3.5 \mathrm{~cm}$. We observed a well-defined, ovoid, firm mass measuring $2.5 \times 2.1 \times 2.0 \mathrm{~cm}$ in the splenic parenchyma (Figure 2) . Histological results revealed a poorly differentiated carcinoma consistent with metastatic carcinoma of the ovary. The CA-125 level dropped to $34.90 \mathrm{U} / \mathrm{ml}$ after the operation. The patient received adjuvant systemic chemotherapy with belotecan and platinum and was disease free for 42 months.

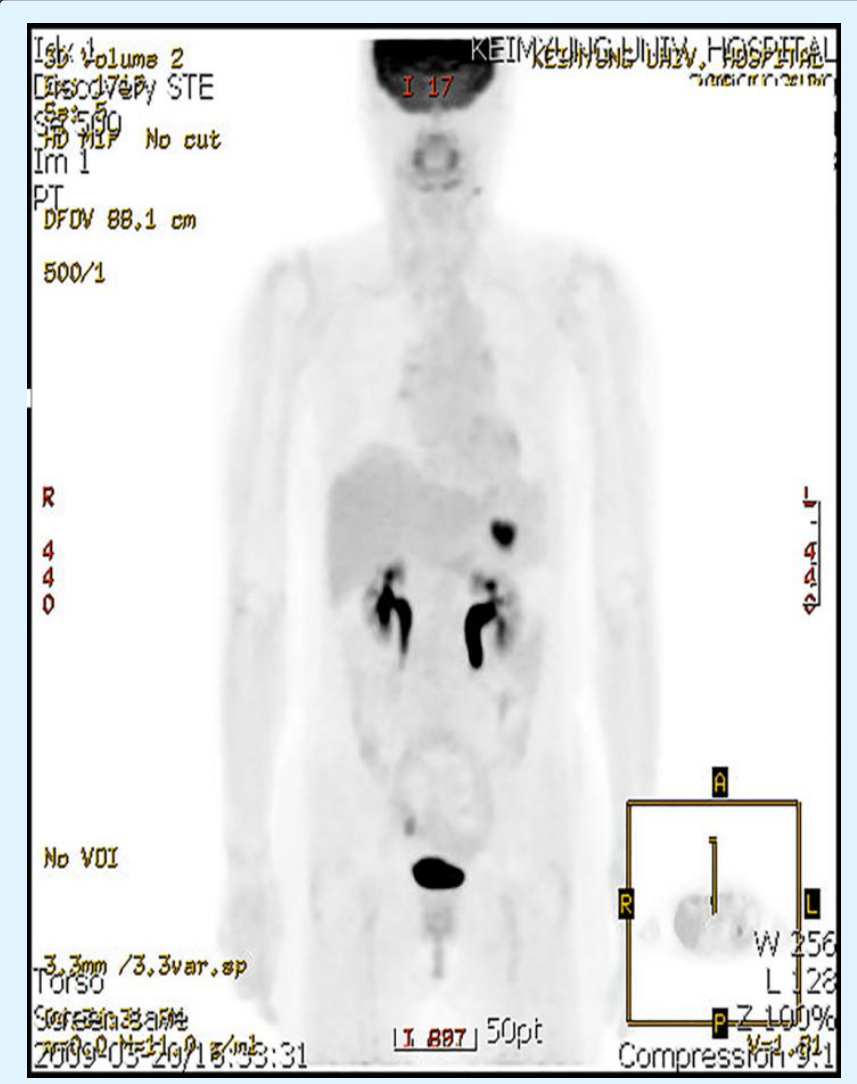

Figure1: PET scan revealed a newly formed, large, well-defined, round, intense hypermetabolic lesion at the inverted splenichilum.

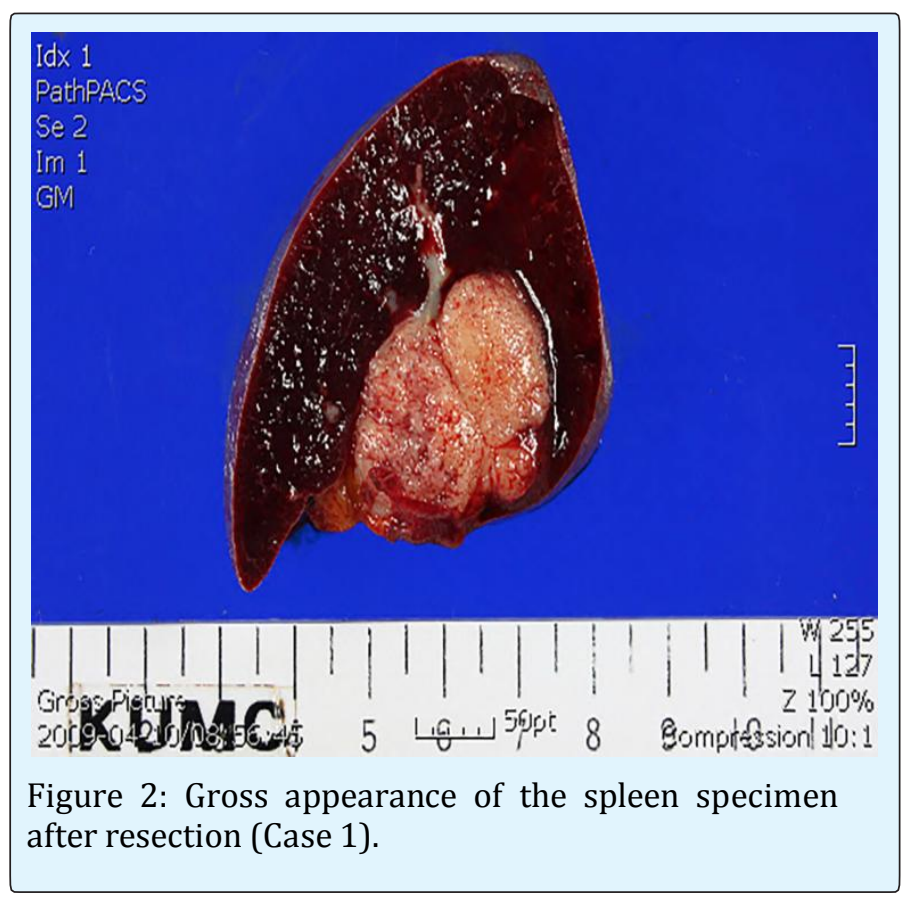

\section{Case 2}

A 58-year-old woman was admitted to our department due to a solitary splenic mass. Two years prior to her admission, she had undergone a cytoreductive operation that included hysterectomy, bilateral salpingooophorectomy, partial omentectomy, appendectomy, and lymph node dissection. Histopathological examination revealed ovarian papillary serous cystadenocarcinoma, stage FIGO IIIc. Following the surgery, she received six cycles of adjuvant chemotherapy with paclitaxel and carboplatin and three cycles of consolidation chemotherapy with the same regimen. Follow-up abdominal computed tomography (CT) showed a newly visible low-attenuating nodule in the spleen (Figure 3), and laparoscopic splenectomy was performed. Macroscopically the spleen was $60 \mathrm{~g}$ with a focal fibrotic and nodular surface (Figure 4). Histological results revealed a metastatic adenocarcinoma, primary in the ovary. She was discharged on postoperative day 8 without any postoperative complications. She received adjuvant systemic chemotherapy with paclitaxel and carboplatin and was disease free for 24 months. 


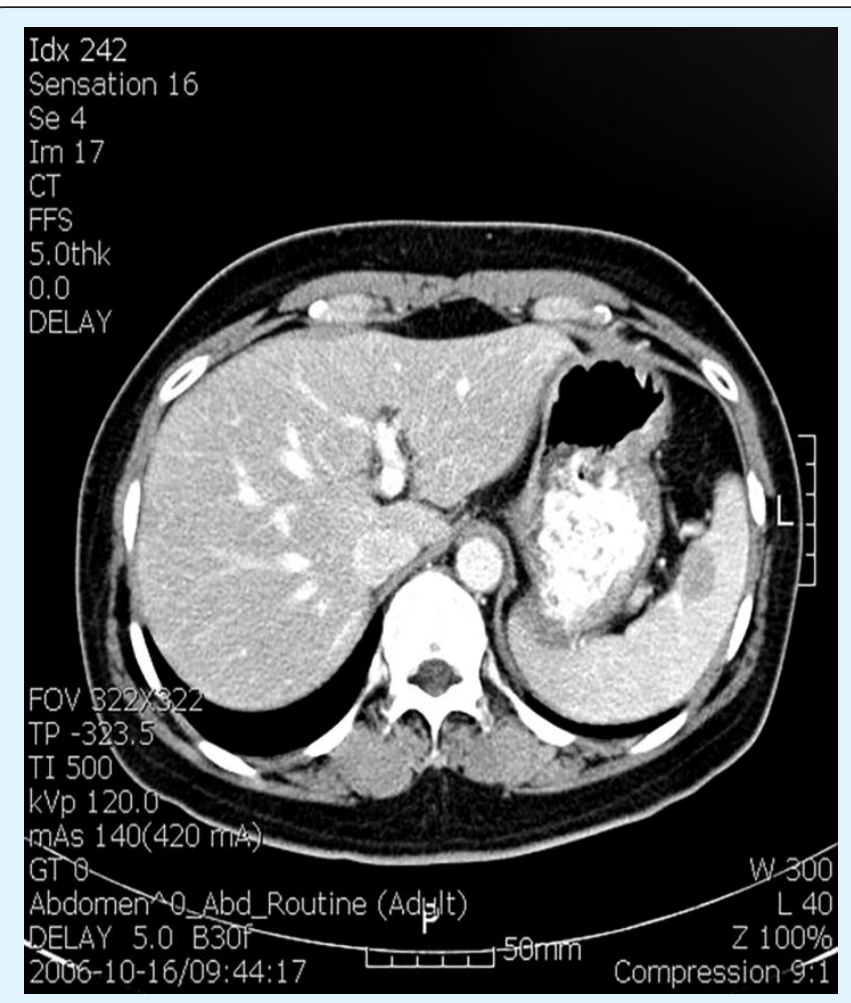

Figure 3: CT showed visible low-attenuating nodules in the spleen.

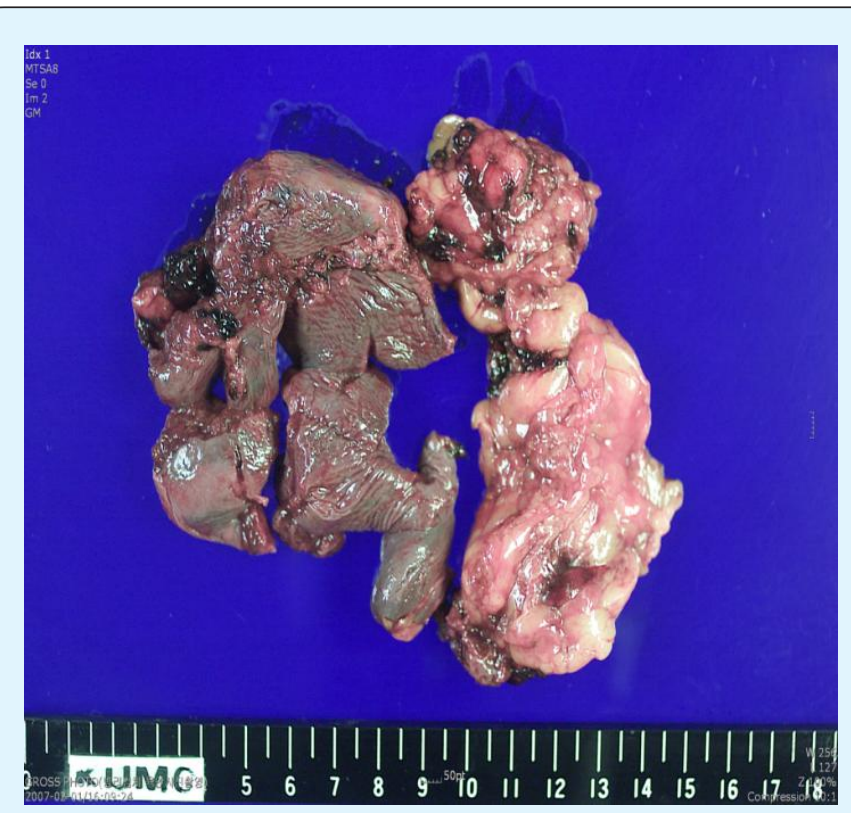

Figure 4: Gross appearance of the spleen specimen after resection (Case 2).

\section{Case 3}

An 82-year-old woman came to our department's outpatient clinic due to lower abdominal discomfort and aggravated abdominal distension, which had been ongoing for 2 months. The patient underwent a cytoreductive operation that included total hysterectomy, bilateral salpingo-oophorectomy, total omentectomy, appendectomy, and lymph node dissection. Histopathological examination revealed poorly differentiated serous adenocarcinoma (Grade 3) with a focal transitional cell carcinoma pattern. She was classified as FIGO stage IIIc and received six cycles of paclitaxel and carboplatin. After completion of chemotherapy, the follow-up examination CT revealed an 11-mm solid nodule in the spleen (Figure 5). Under the impression of isolated splenic metastasis, laparoscopic splenectomy was performed. Her postoperative course was uneventful; however, histopathological evaluation revealed no tumor in the spleen. She has remained alive and disease free for 9 years.

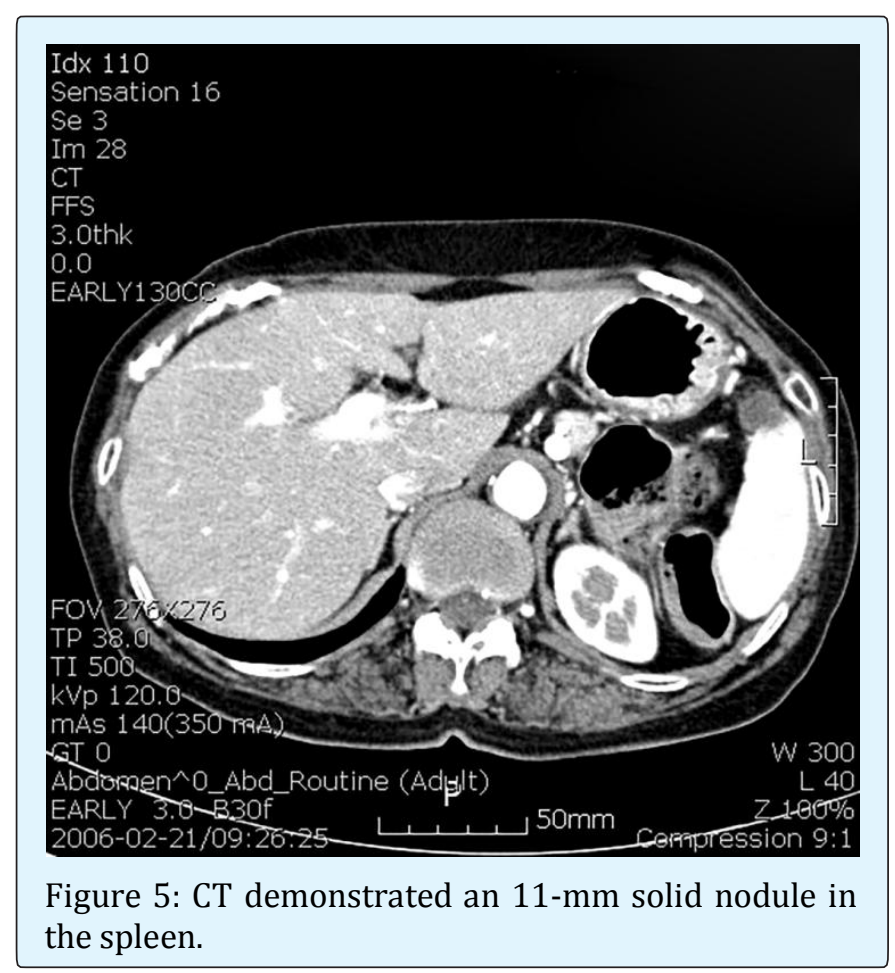

\section{Discussion}

The biological behavior of ovarian carcinoma is markedly different from the classic and well-known pattern of hematogenous metastasis found in most other cancers. Once the cancer cells have detached as single 
cells or clusters from the primary ovarian tumor, it is thought that they metastasize through a passive mechanism, carried by the physiological movement of peritoneal fluid to the peritoneum and omentum. Clinical observation and retrospective clinical studies suggest that serous ovarian carcinomas grow very efficiently within the peritoneal cavity in a pattern of multiple seeding but rarely metastasize to a single organ [2]. This type of carcinoma usually spreads transperitoneally to neighboring organs, such as the rectum, sigmoid colon, and urinary bladder. In addition to direct and peritoneal invasion, lymphatic and hematogenous dissemination are also common. The most common sites of lymph node involvement are intra-abdominal and para-aortic lymph nodes.

Only 36 cases of isolated parenchymal metastasis of ovarian cancer have been reported in the literature. Solitary parenchymal metastases of the spleen are most probably generated through the hematogenic route, which is rare because of several mechanisms, including rhythmic contractions of the splenic capsule, the sharp angle of the splenic artery branching from the celiac artery, lack of afferent lymphatic vessels to the spleen, and mostly the inhibitory effect of the splenic microenvironment on the growth of metastatic cells [3].

The current treatment of advanced ovarian carcinoma relies on aggressive surgical cytoreduction followed by platinum-based chemotherapy. Although the role of secondary cytoreductive surgery in the treatment of disseminated disease remains unclear, patients with solitary metastases seem to have better survival after optimal secondary debulking. Solitary metastases can often be treated successfully with an expectation of prolonged survival. Secondary debulking has been advocated as a means of augmenting the durability of response to subsequent adjuvant chemotherapy [4]. A recent retrospective study by Magrina et al. [5] compared laparotomic, laparoscopic, and robotic surgeries among 52 selected recurrent ovarian cancer patients undergoing secondary cytoreduction. Their study showed that patients localize disease benefit from a laparoscopic or robotic secondary cytoreduction and experience comparable survival, whereas laparotomy is the preferred treatment for patients with widespread peritoneal implants, multiple sites of recurrence, or extensive adhesions. For an isolated splenic metastasis, most reported cases are of open laparotomy [6,7]; however, laparoscopic splenectomy can provide several advantages over laparotomy, such as a shorter operation time, better visualization, less blood loss, shorter hospital stay, and the possibility of an earlier initiation of postoperative chemotherapy. Three cases were included in this case reports with institutional ethics board approval (No. 2015-07-025). In our third case, a newly developed, solitary, metastatic spleen lesion was observed in preoperative imaging, but biopsy results showed no tumor. This is a good example of the laparoscopic approach compared to open laparotomy as the patient will not receive any unnecessary adjuvant chemotherapy. Consequently, laparoscopic approach can offer better postoperative quality of life from a faster recovery without compromising patient survival. When an isolated splenic lesion is detected during follow-up imaging, the laparoscopic approach can be an effective surgical method of diagnosis and treatment at the same time without increasing morbidity. In a short period of time, it can provide more guidance to the next therapeutic approach. We suggest that laparoscopic splenectomy can be a proper and feasible therapeutic modality for isolated splenic metastasis.

\section{Conclusion}

The presented case reports suggest that laparoscopic splenectomy should be considered when isolated splenic parenchymal metastasis is suspected in recurrent ovarian cancer. Laparoscopic splenectomy is feasible with less morbidity, and patients present a prolonged disease-free survival with better postoperative quality of life from a faster recovery compared to laparotomy. Further serial cases should be reported to more thoroughly determine the effectiveness of laparoscopic splenectomy and to suggest the next therapeutic modality for isolated splenic metastasis in recurrent ovarian cancer.

\section{Acknowledgment}

This work was supported by the Scholar Research Grant of Keimyung University in 2015.

\section{References}

1. Eisenkop SM, Spirtos NM (2001) The Clinical Significance of Occult Macroscopically Positive Retroperitoneal Nodes in Patients with Epithelial Ovarian Cancer. Gynecologic Oncology 82(1): 143149.

2. Lengyel E (2010) Ovarian Cancer Development and Metastasis. Am J Pathol 177(3): 1053-1064. 
3. Comperat E, Bardier-Dupas A, Camparo P, Capron F, Charlotte F (2007) Splenic metastases: clinicopathologic presentation, differential diagnosis, and pathogenesis. Arch Pathol Lab Med 131(6): 965969.

4. Bristow RE, Puri I, Chi DS (2009) Cytoreductive surgery for recurrent ovarian cancer: A metaanalysis. Gynecologic Oncology 112(1): 265-274.

5. Magrina JF, Cetta RL, Chang YH, Guevara G, Magtibaby PM (2013) Analysis of secondary cytoreduction for recurrent ovarian cancer by robotics, laparoscopy, and laparotomy. Gynecol Oncol 129(2): 336-340.
6. Tserkezoglou A, Kontou S, Hatjieleftheriou G, Nikolaidou ME, Plataniotis G, et al. (2005) Solitary Parenchymal Splenic Recurrence of Ovarian Adenocarcinoma: A Case Report and Review of the Literature. Anticancer Res 25(2B): 1471-1476.

7. Karni D, Kopelman D, Abu Hatoum O (2014) Solitary splenic metastasis of ovarian carcinoma: a case report. J Med Case Rep 8: 154-157. 